\title{
Nonlinear Adaptive Bilateral Control of Teleoperation Systems with Uncertain Dynamics and Kinematics
}

\author{
X. Liu, M. Tavakoli, and Q. Huang
}

\begin{abstract}
Research so far on adaptive bilateral control of master-slave teleoperation systems considers dynamic uncertainties but stops short of considering kinematic uncertainties. However, when picking up objects of unknown lengths, orientations and gripping points, the overall kinematics of a robot in the teleoperation system becomes uncertain. Therefore, new controllers are required that can guarantee the stability and motion tracking performance of the system in the presence of both dynamic and kinematic uncertainties in the master and the slave robots. In this paper, first the uncertain dynamics of the human operator and the environment are incorporated into the dynamics of the master and the slave, respectively. Then, for a teleoperation system with uncertain dynamics and kinematics, nonlinear adaptive controllers are designed for both the master and the slave. The controllers do not need exact knowledge of the dynamics of the master, the slave, the operator, or the environment, or of the kinematics of the master or the slave. The stability and position tracking convergence of the entire teleoperation system are studied. The validity of the theoretical results is verified by simulations.
\end{abstract}

\section{INTRODUCTION}

$\mathrm{T}$ eleoperation systems enable humans to reach and interact with environments that are too remote (e.g., outer space), too confined (e.g., human body cavity), or too hazardous (e.g., a nuclear waste site or mine field) for the human to be in. A typical teleoperation system consists of a local robot (master), a teleoperated robot (slave), a human operator, and an environment. The human operator applies forces on the master to remotely control the position of the slave in order to perform a task such as grasping an object in the remote environment. In such a system, force reflection involves capturing the robot-environment interaction and displaying it to the operator. If the slave exactly reproduces the master's position trajectory for the environment and the master accurately displays the slave-environment contact force to the human, the teleoperation system is said be fully transparent. For a review of teleoperation control approaches, see the surveys [1]-[2].

Most teleoperation controllers assume perfect knowledge of the master and the slave impedances. In a teleoperation

Manuscript received March 6, 2010. This research was supported by the Natural Sciences and Engineering Research Council (NSERC) of Canada under Grants RGPIN-372042 and EQPEQ-375712 and by the China Scholarship Council (CSC) under Grant [2009]3012.

X. Liu and Q. Huang are with the School of Automation Engineering, University of Electronic Science and Technology of China, Chengdu, Sichuan, 610054 China. X. Liu is now a visiting PhD student in the Department of Electrical and Computer Engineering, University of Alberta, Edmonton, Alberta, T6G 2V4 Canada. (e-mail: xia8@ualberta.ca).

M. Tavakoli is with the Department of Electrical and Computer Engineering, University of Alberta, Edmonton, Alberta, T6G 2V4 Canada. (e-mail: tavakoli@ece.ualberta.ca). system, however, perfect knowledge of the master and the slave cannot be obtained in practice due to model uncertainties and/or unmodelled dynamics. Besides, the closed-loop stability is affected by the operator and the environment dynamics, which are both uncertain.

Zaad and Salcudean [3] combined the dynamics of the environment and slave in the design of an adaptive controller for the slave used to mitigate parametric uncertainties in the environment model. Lee and Chung [4] designed an adaptive control scheme for teleoperation systems with parametric uncertainties in the slave and environment dynamics. Shi et al. [5] developed several adaptive control schemes for teleoperation systems with parametric uncertainties in the slave and with environments of different types: unknown constant parameters, jumping parameters, and smooth timevarying parameters. Fite et al. [6] proposed a predictive adaptive controller to extend the Smith predictor concept to the force-position teleoperation control architecture to cope with the effect of time delays and maintain transparency independent of parametric uncertainties in the environment. In all of these, (a) the dynamics of the master and the slave were assumed to be linear whereas multi-DOF robots are typically nonlinear (note that applying linear approaches to nonlinear teleoperation systems results in degraded performance), and (b) dynamic uncertainties of the slave and/or the environment were considered yet those of the master and the operator were ignored. In other words, an adaptive controller was only designed for the slave side.

For adaptive teleoperation control involving nonlinear models of the master and the slave, Ryu and Kwon [7] did not include the environment's and the human's uncertain parameters in the adaption and achieved full transparency. Hung et al. [8] designed adaptive controllers for both the master and the slave by introducing a virtual master to achieve stability and motion tracking performance. Chopra et al. [9] proposed an adaptive controller for a teleoperator with time delay to ensure synchronization of positions and velocities of the master and the slave in free motion. Nuño et al. [10] showed that Chopra's scheme was applicable only to systems without gravity and, to overcome this, a new adaptive controller that replaces the positions and velocities by their errors was proposed. The limitation of all of the above is that they only consider the uncertain dynamics of the master and the slave, and do not consider the uncertainties introduced by the human and the environment.

For nonlinear master and slave models and linear human and environment models - all subject to parametric uncertainties - Zhu and Salcudean [11], Sirouspour and Setoodeh [12], and Malysz and Sirouspour [13] proposed adaptive teleoperation controllers to enforce linear scalar- 
type position/force mappings between the human and the environment. Separate adaptive laws were designed for the master side and for the slave side. Nevertheless, this and other adaptive schemes did not account for uncertain kinematics of the master and the slave robots.

This leads us to several open problems in the development of teleoperation control laws. Note that the kinematic parameters of robots are quite hard to measure [14]. Moreover, the tool-induced kinematic uncertainties add to the complexity. Thus, when a robot picks up objects of different lengths, unknown orientations and gripping points, the overall kinematics is affected. The result is that the robot is no longer able to precisely manipulate a tool to a desired position. A question that naturally follows is how to achieve stabilizing and high-performing teleoperation in the face of both dynamic and kinematic uncertainties?

This paper proposes nonlinear adaptive controllers for teleoperation systems with uncertain dynamics and kinematics. The proposed teleoperation control laws are inspired by those proposed by Cheah et al. [15]-[17]. It must be noted, however, that the results in [15] and [16] deal with motion control of a single robot in free motion. Also, in [17], both position and force sensors are needed for motion and force tracking in a single robot, which is expensive to realize in practice. The major contribution of this paper is incorporating the parameter-uncertain dynamics of the human operator and the environment into the parameteruncertain dynamics of the master and the slave in the joint space, and extending the control approach for a single robot to a teleoperation system. In doing so, we also assume parametric uncertainties in the master and slave kinematics.

The rest of this paper is organized as follows. In Section II, we first introduce the dynamic and kinematic models of a teleoperation system. Next, we assume parameter-uncertain LTI models for the operator and the environment in the task space and convert them into the joint space, so that we can incorporate them into the master and the slave dynamics, respectively. In Section III, nonlinear adaptive controllers are designed for the master and slave sides. The stability and motion tracking convergence of the teleoperation system are studied. Simulation results in Section IV show that the slave can follow the position of the master despite all uncertainties in dynamics and kinematics. Besides, the force tracking error between the master and the slave is bounded. The paper is concluded in Section V.

\section{DYNAMIC AND KinEMATIC MODELS OF TELEOPERATOR}

\section{A. Dynamics of Master and Slave in Joint Space}

In the absence of friction and other disturbances, when interacting with a human and an environment, the jointspace nonlinear dynamic models for an n-DOF master robot and an n-DOF slave robot can be described as [18]:

$$
\mathbf{M}_{\mathrm{qm}}\left(\mathbf{q}_{\mathrm{m}}\right) \ddot{\mathbf{q}}_{\mathrm{m}}+\mathbf{C}_{\mathrm{qm}}\left(\mathbf{q}_{\mathrm{m}}, \dot{\mathbf{q}}_{\mathrm{m}}\right) \dot{\mathbf{q}}_{\mathrm{m}}+\mathbf{G}_{\mathrm{qm}}\left(\mathbf{q}_{\mathrm{m}}\right)
$$

$$
\begin{gathered}
=\boldsymbol{\tau}_{\mathrm{m}}+\mathbf{J}_{\mathrm{m}}^{\mathrm{T}}\left(\mathbf{q}_{\mathrm{m}}\right) \mathbf{f}_{\mathrm{h}} \\
\mathbf{M}_{\mathrm{qs}}\left(\mathbf{q}_{\mathrm{s}}\right) \ddot{\mathbf{q}}_{\mathrm{s}}+\mathbf{C}_{\mathrm{qs}}\left(\mathbf{q}_{\mathrm{s}}, \dot{\mathbf{q}}_{\mathrm{s}}\right) \dot{\mathbf{q}}_{\mathrm{s}}+\mathbf{G}_{\mathrm{qs}}\left(\mathbf{q}_{\mathrm{s}}\right) \\
=\boldsymbol{\tau}_{\mathrm{s}}-\mathbf{J}_{\mathrm{s}}^{\mathrm{T}}\left(\mathbf{q}_{\mathrm{s}}\right) \mathbf{f}_{\mathrm{e}}
\end{gathered}
$$

where $\mathbf{q}_{\mathrm{m}}$ and $\mathbf{q}_{\mathrm{s}} \in \mathrm{R}^{\mathrm{n} \times 1}$ are joint angle positions, $\mathbf{M}_{\mathrm{qm}}\left(\mathbf{q}_{\mathrm{m}}\right)$ and $\mathbf{M}_{\mathrm{qs}}\left(\mathbf{q}_{\mathrm{s}}\right) \in \mathrm{R}^{\mathrm{n} \times \mathrm{n}}$ are symmetric positivedefinite inertia matrices, $\mathbf{C}_{\mathrm{qm}}\left(\mathbf{q}_{\mathrm{m}}, \dot{\mathbf{q}}_{\mathrm{m}}\right)$ and $\mathbf{C}_{\mathrm{qs}}\left(\mathbf{q}_{\mathrm{s}}, \dot{\mathbf{q}}_{\mathrm{s}}\right) \in$ $\mathrm{R}^{\mathrm{n} \times \mathrm{n}}$ are matrices representing Coriolis and centrifugal terms, $\mathbf{G}_{\mathrm{qm}}\left(\mathbf{q}_{\mathrm{m}}\right)$ and $\mathbf{G}_{\mathrm{qs}}\left(\mathbf{q}_{\mathrm{s}}\right) \in \mathrm{R}^{\mathrm{n} \times 1}$ represent gravity terms, $\boldsymbol{\tau}_{\mathrm{m}}$ and $\boldsymbol{\tau}_{\mathrm{s}} \in \mathrm{R}^{\mathrm{n} \times 1}$ are control inputs (torques), $\mathbf{f}_{\mathrm{h}} \in$ $\mathrm{R}^{\mathrm{n} \times 1}$ denotes the force the operator applies to the master, $\mathbf{f}_{\mathrm{e}} \in \mathrm{R}^{\mathrm{n} \times 1}$ is the force the environment applies to the slave, and $\mathbf{J}_{\mathrm{m}}\left(\mathbf{q}_{\mathrm{m}}\right)$ and $\mathbf{J}_{\mathrm{s}}\left(\mathbf{q}_{\mathrm{s}}\right) \in \mathrm{R}^{\mathrm{n} \times \mathrm{n}}$ are the Jacobian matrices for the master and the slave, respectively.

Dynamics (1)-(2) have some fundamental properties [18]:

Property 1. The left sides of (1) and (2) are linear in a set of dynamic parameters $\boldsymbol{\theta}_{\mathrm{d}}=\left(\theta_{\mathrm{d} 1}, \ldots, \theta_{\mathrm{dp}}\right)^{\mathrm{T}}$ as

$$
\mathbf{M}(\mathbf{q}) \ddot{\mathbf{q}}+\mathbf{C}(\mathbf{q}, \dot{\mathbf{q}}) \dot{\mathbf{q}}+\mathbf{G}(\mathbf{q})=\mathbf{Y}_{\mathrm{d}}(\mathbf{q}, \dot{\mathbf{q}}, \ddot{\mathbf{q}}) \boldsymbol{\theta}_{\mathrm{d}}
$$

where $\mathbf{Y}_{\mathrm{d}}(\mathbf{q}, \dot{\mathbf{q}}) \in \mathrm{R}^{\mathrm{n} \times \mathrm{p}}$ is the dynamic regressor matrix.

Property 2. The matrix $\dot{\mathbf{M}}(\mathbf{q})-2 \mathbf{C}(\mathbf{q}, \dot{\mathbf{q}})$ is skew-symmetric

$$
\text { i.e., } \boldsymbol{\zeta}^{\mathrm{T}}(\dot{\mathbf{M}}(\mathbf{q})-2 \mathbf{C}(\mathbf{q}, \dot{\mathbf{q}})) \zeta=0, \quad \forall \zeta \in \mathrm{R}^{\mathrm{n} \times 1} .
$$

\section{B. Kinematics of Master and Slave}

The generalized end-effector positions $\mathbf{x}_{\mathrm{m}}$ and $\mathbf{x}_{\mathrm{s}} \in \mathrm{R}^{6 \times 1}$ of the master and the slave can be expressed as [18],

$$
\mathbf{x}_{\mathrm{m}}=\mathbf{h}_{\mathrm{m}}\left(\mathbf{q}_{\mathrm{m}}\right), \mathbf{x}_{\mathrm{s}}=\mathbf{h}_{\mathrm{s}}\left(\mathbf{q}_{\mathrm{s}}\right)
$$

where $\mathbf{h}(.) \in \mathrm{R}^{\mathrm{n}} \rightarrow \mathrm{R}^{6}$ is a nonlinear transformation describing the relation between the joint-space and the taskspace positions. The Jacobian-based relationships between task-space and joint-space velocities are

$$
\dot{\mathbf{x}}_{\mathrm{m}}=\mathrm{J}_{\mathrm{m}}\left(\mathbf{q}_{\mathrm{m}}\right) \dot{\mathbf{q}}_{\mathrm{m}}, \dot{\mathbf{x}}_{\mathrm{s}}=\mathrm{J}_{\mathrm{s}}\left(\mathbf{q}_{\mathrm{s}}\right) \dot{\mathbf{q}}_{\mathrm{s}}
$$

Differentiating (4) with respect to time yields

$$
\begin{aligned}
& \ddot{\mathbf{x}}_{\mathrm{m}}=\dot{\mathbf{J}}_{\mathrm{m}}\left(\mathbf{q}_{\mathrm{m}}\right) \dot{\mathbf{q}}_{\mathrm{m}}+\mathbf{J}_{\mathrm{m}}\left(\mathbf{q}_{\mathrm{m}}\right) \ddot{\mathbf{q}}_{\mathrm{m}} \\
& \ddot{\mathbf{x}}_{\mathrm{s}}=\dot{\mathbf{J}}_{\mathrm{s}}\left(\mathbf{q}_{\mathrm{s}}\right) \dot{\mathbf{q}}_{\mathrm{s}}+\mathbf{J}_{\mathrm{s}}\left(\mathbf{q}_{\mathrm{s}}\right) \ddot{\mathbf{q}}_{\mathrm{s}}
\end{aligned}
$$

Property 3. The right side of (4) is linear in a set of kinematic parameters [15]-[17] $\boldsymbol{\theta}_{\mathrm{mk}}=\left(\theta_{\mathrm{k} 1}, \ldots, \theta_{\mathrm{kw}_{1}}\right)^{\mathrm{T}}$ and $\boldsymbol{\theta}_{\mathrm{sk}}=\left(\theta_{\mathrm{k} 1}, \ldots, \theta_{\mathrm{kw}_{2}}\right)^{\mathrm{T}}$ such that

$$
\begin{aligned}
& \dot{\mathbf{x}}_{\mathrm{m}}=\mathrm{J}_{\mathrm{m}}\left(\mathbf{q}_{\mathrm{m}}\right) \dot{\mathbf{q}}_{\mathrm{m}}=\mathbf{Y}_{\mathrm{mk}}\left(\mathbf{q}_{\mathrm{m}}, \dot{\mathbf{q}}_{\mathrm{m}}\right) \boldsymbol{\theta}_{\mathrm{mk}} \\
& \dot{\mathbf{x}}_{\mathrm{s}}=\mathbf{J}_{\mathrm{s}}\left(\mathbf{q}_{\mathrm{s}}\right) \dot{\mathbf{q}}_{\mathrm{s}}=\mathbf{Y}_{\mathrm{sk}}\left(\mathbf{q}_{\mathrm{s}}, \dot{\mathbf{q}}_{\mathrm{s}}\right) \boldsymbol{\theta}_{\mathrm{sk}}
\end{aligned}
$$

where $\mathbf{Y}_{\mathrm{mk}}\left(\mathbf{q}_{\mathrm{m}}, \dot{\mathbf{q}}_{\mathrm{m}}\right) \in \mathrm{R}^{6 \times \mathrm{w}_{1}}$ and $\mathbf{Y}_{\mathrm{sk}}\left(\mathbf{q}_{\mathrm{s}}, \dot{\mathbf{q}}_{\mathrm{s}}\right) \in \mathrm{R}^{6 \times \mathrm{w}_{2}}$ are the kinematic regressor matrices.

\section{Dynamics of Operator and Environment in Task Space}

The dynamics of the human operator and the environment are naturally specified in the task space as it is the space in which they make contact with the master and the slave 
robots. In this paper, second-order LTI models are used for the human and the environment. Such models have been successfully used by other researchers (see [11]-[13]).

$$
\begin{aligned}
& \mathbf{f}_{\mathrm{h}}=\mathbf{f}_{\mathrm{h}}^{*}-\left(\mathbf{M}_{\mathrm{h}} \ddot{\mathbf{x}}_{\mathrm{m}}+\mathbf{B}_{\mathrm{h}} \dot{\mathbf{x}}_{\mathrm{m}}+\mathbf{K}_{\mathrm{h}} \mathbf{x}_{\mathrm{m}}\right) \\
& \mathbf{f}_{\mathrm{e}}=\mathbf{f}_{\mathrm{e}}^{*}+\mathbf{M}_{\mathrm{e}} \ddot{\mathbf{x}}_{\mathrm{s}}+\mathbf{B}_{\mathrm{e}} \dot{\mathbf{x}}_{\mathrm{s}}+\mathbf{K}_{\mathrm{e}} \mathbf{x}_{\mathrm{s}}
\end{aligned}
$$

where $\mathbf{M}_{\mathrm{h}}, \mathbf{M}_{\mathrm{e}}, \mathbf{B}_{\mathrm{h}}, \mathbf{B}_{\mathrm{e}}, \mathbf{K}_{\mathrm{h}}$ and $\mathbf{K}_{\mathrm{e}}$ are constant, symmetric and positive matrices in $\mathrm{R}^{6 \times 6}$ corresponding to the mass, damping, and stiffness of the operator's hand and the environment, respectively. Here, $\mathbf{f}_{\mathrm{h}}^{*}$ is the human exogenous force and $\mathbf{f}_{\mathrm{e}}^{*}$ is the environment exogenous force.

\section{End-to-end Model of Teleoperation System in Joint Space}

To facilitate the teleoperation control design, the dynamics of the human and the environment are transformed from task to joint space and incorporated into the dynamics of the master and the slave. Substituting (3)-(6) into (7)-(8), the joint-space models of the human and the environment are

$$
\begin{aligned}
\mathbf{f}_{\mathrm{h}}= & \mathbf{f}_{\mathrm{h}}^{*}-\mathbf{M}_{\mathrm{h}} \mathbf{J}_{\mathrm{m}}\left(\mathbf{q}_{\mathrm{m}}\right) \ddot{\mathbf{q}}_{\mathrm{m}}-\left(\mathbf{B}_{\mathrm{h}} \mathbf{J}_{\mathrm{m}}\left(\mathbf{q}_{\mathrm{m}}\right)+\mathbf{M}_{\mathrm{h}} \dot{\mathbf{J}}_{\mathrm{m}}\left(\mathbf{q}_{\mathrm{m}}\right)\right) \dot{\mathbf{q}}_{\mathrm{m}} \\
& -\mathbf{K}_{\mathrm{h}} \mathbf{h}_{\mathrm{m}}\left(\mathbf{q}_{\mathrm{m}}\right) \\
\mathbf{f}_{\mathrm{e}}= & \mathbf{f}_{\mathrm{e}}^{*}+\mathbf{M}_{\mathrm{e}} \mathbf{J}_{\mathrm{s}}\left(\mathbf{q}_{\mathrm{s}}\right) \ddot{\mathbf{q}}_{\mathrm{s}}+\left(\mathbf{B}_{\mathrm{e}} \mathbf{J}_{\mathrm{s}}\left(\mathbf{q}_{\mathrm{s}}\right)+\mathbf{M}_{\mathrm{e}} \dot{\mathbf{J}}_{\mathrm{s}}\left(\mathbf{q}_{\mathrm{s}}\right)\right) \dot{\mathbf{q}}_{\mathrm{s}} \\
& +\mathbf{K}_{\mathrm{e}} \mathbf{h}_{\mathrm{s}}\left(\mathbf{q}_{\mathrm{s}}\right)
\end{aligned}
$$

Multiplying both sides of (9)-(10) by $\mathbf{J}_{\mathrm{m}}^{\mathrm{T}}\left(\mathbf{q}_{\mathrm{m}}\right)$ and $\mathbf{J}_{\mathrm{s}}^{\mathrm{T}}\left(\mathbf{q}_{\mathrm{s}}\right)$, respectively, and substituting into (1)-(2), a combined model for the master/operator system and a combined model for the slave/environment system are obtained as

$$
\begin{aligned}
& \mathbf{M}_{\mathrm{m}}\left(\mathbf{q}_{\mathrm{m}}\right) \ddot{\mathbf{q}}_{\mathrm{m}}+\mathbf{C}_{\mathrm{m}}\left(\mathbf{q}_{\mathrm{m}}, \dot{\mathbf{q}}_{\mathrm{m}}\right) \dot{\mathbf{q}}_{\mathrm{m}}+\mathbf{G}_{\mathrm{m}}\left(\mathbf{q}_{\mathrm{m}}\right)=\boldsymbol{\tau}_{\mathrm{m}} \\
& \mathbf{M}_{\mathrm{s}}\left(\mathbf{q}_{\mathrm{s}}\right) \ddot{\mathbf{q}}_{\mathrm{s}}+\mathbf{C}_{\mathrm{s}}\left(\mathbf{q}_{\mathrm{s}}, \dot{\mathbf{q}}_{\mathrm{s}}\right) \dot{\mathbf{q}}_{\mathrm{s}}+\mathbf{G}_{\mathrm{s}}\left(\mathbf{q}_{\mathrm{s}}\right)=\boldsymbol{\tau}_{\mathrm{s}}
\end{aligned}
$$

where

$$
\begin{gathered}
\mathbf{M}_{\mathrm{m}}\left(\mathbf{q}_{\mathrm{m}}\right)=\mathbf{M}_{\mathrm{qm}}\left(\mathbf{q}_{\mathrm{m}}\right)+\mathbf{J}_{\mathrm{m}}^{\mathrm{T}}\left(\mathbf{q}_{\mathrm{m}}\right) \mathbf{M}_{\mathrm{h}} \mathbf{J}_{\mathrm{m}}\left(\mathbf{q}_{\mathrm{m}}\right) \\
\mathbf{C}_{\mathrm{m}}\left(\mathbf{q}_{\mathrm{m}}, \dot{\mathbf{q}}_{\mathrm{m}}\right)=\mathbf{C}_{\mathrm{qm}}\left(\mathbf{q}_{\mathrm{m}}, \dot{\mathbf{q}}_{\mathrm{m}}\right)+\mathbf{J}_{\mathrm{m}}^{\mathrm{T}}\left(\mathbf{q}_{\mathrm{m}}\right) \mathbf{B}_{\mathrm{h}} \mathbf{J}_{\mathrm{m}}\left(\mathbf{q}_{\mathrm{m}}\right) \\
+\mathbf{J}_{\mathrm{m}}^{\mathrm{T}}\left(\mathbf{q}_{\mathrm{m}}\right) \mathbf{M}_{\mathrm{h}} \dot{\mathbf{J}}_{\mathrm{m}}\left(\mathbf{q}_{\mathrm{m}}\right) \\
\mathbf{G}_{\mathrm{m}}\left(\mathbf{q}_{\mathrm{m}}\right)=\mathbf{G}_{\mathrm{qm}}\left(\mathbf{q}_{\mathrm{m}}\right)+\mathbf{J}_{\mathrm{m}}^{\mathrm{T}}\left(\mathbf{q}_{\mathrm{m}}\right) \mathbf{K}_{\mathrm{h}} \mathbf{h}_{\mathrm{m}}\left(\mathbf{q}_{\mathrm{m}}\right)-\mathbf{J}_{\mathrm{m}}^{\mathrm{T}}\left(\mathbf{q}_{\mathrm{m}}\right) \mathbf{f}_{\mathrm{h}}^{*} \\
\mathbf{M}_{\mathrm{s}}\left(\mathbf{q}_{\mathrm{s}}\right)=\mathbf{M}_{\mathrm{qs}}\left(\mathbf{q}_{\mathrm{s}}\right)+\mathbf{J}_{\mathrm{s}}^{\mathrm{T}}\left(\mathbf{q}_{\mathrm{s}}\right) \mathbf{M}_{\mathrm{e}} \mathbf{J}_{\mathrm{s}}\left(\mathbf{q}_{\mathrm{s}}\right) \\
\mathbf{C}_{\mathrm{s}}\left(\mathbf{q}_{\mathrm{s}}, \dot{\mathbf{q}}_{\mathrm{s}}\right)=\mathbf{C}_{\mathrm{qs}}\left(\mathbf{q}_{\mathrm{s}}, \dot{\mathbf{q}}_{\mathrm{s}}\right)+\mathbf{J}_{\mathrm{s}}^{\mathrm{T}}\left(\mathbf{q}_{\mathrm{s}}\right) \mathbf{B}_{\mathrm{e}} \mathbf{J}_{\mathrm{s}}\left(\mathbf{q}_{\mathrm{s}}\right) \\
\quad+\mathbf{J}_{\mathrm{s}}^{\mathrm{T}}\left(\mathbf{q}_{\mathrm{s}}\right) \mathbf{M}_{\mathrm{e}} \dot{\mathbf{J}}_{\mathrm{s}}\left(\mathbf{q}_{\mathrm{s}}\right) \\
\mathbf{G}_{\mathrm{s}}\left(\mathbf{q}_{\mathrm{s}}\right)=\mathbf{G}_{\mathrm{qs}}\left(\mathbf{q}_{\mathrm{s}}\right)+\mathbf{J}_{\mathrm{s}}^{\mathrm{T}}\left(\mathbf{q}_{\mathrm{s}}\right) \mathbf{K}_{\mathrm{e}} \mathbf{h}_{\mathrm{s}}\left(\mathbf{q}_{\mathrm{s}}\right)+\mathbf{J}_{\mathrm{s}}^{\mathrm{T}}\left(\mathbf{q}_{\mathrm{s}}\right) \mathbf{f}_{\mathrm{e}}^{*}
\end{gathered}
$$

It should be noted that, Property 1 still holds for the left sides of (11)-(12), while Property 2 does not hold for the new inertia and Coriolis/centrifugal matrices. Instead, a new property is introduced below.

Property 4. For $\forall \zeta \in \mathrm{R}^{6 \times 1}$, we have

$$
\boldsymbol{\zeta}^{\mathrm{T}}\left(\dot{\mathbf{M}}_{\mathrm{m}}\left(\mathbf{q}_{\mathrm{m}}\right)-2 \mathbf{C}_{\mathrm{m}}\left(\mathbf{q}_{\mathrm{m}}, \dot{\mathbf{q}}_{\mathrm{m}}\right)\right) \boldsymbol{\zeta}
$$

and

$$
=-2 \boldsymbol{\zeta}^{\mathrm{T}}\left(\mathbf{J}_{\mathrm{m}}^{\mathrm{T}}\left(\mathbf{q}_{\mathrm{m}}\right) \mathbf{B}_{\mathrm{h}} \mathbf{J}_{\mathrm{m}}\left(\mathbf{q}_{\mathrm{m}}\right)\right) \boldsymbol{\zeta}
$$

$$
\begin{aligned}
& \boldsymbol{\zeta}^{\mathrm{T}}\left(\dot{\mathbf{M}}_{\mathrm{s}}\left(\mathbf{q}_{\mathrm{s}}\right)-2 \mathbf{C}_{\mathrm{s}}\left(\mathbf{q}_{\mathrm{s}}, \dot{\mathbf{q}}_{\mathrm{s}}\right)\right) \boldsymbol{\zeta} \\
= & -2 \boldsymbol{\zeta}^{\mathrm{T}}\left(\mathbf{J}_{\mathrm{s}}^{\mathrm{T}}\left(\mathbf{q}_{\mathrm{s}}\right) \mathbf{B}_{\mathrm{e}} \mathbf{J}_{\mathrm{s}}\left(\mathbf{q}_{\mathrm{s}}\right)\right) \boldsymbol{\zeta}
\end{aligned}
$$

Remark 1: Incorporating the operator's dynamics into the master and the environment's dynamics into the slave was done in [11]-[13]. However, the main difference in our work is that we transform the dynamics of the operator and the environment from task space into joint space, so that the dynamics of the master, slave, operator and environment can be unified in the joint space for adaptive control synthesis.

\section{Design OF NONLINEAR ADAPTIVE CONTROLlER}

Various adaptive controllers for a single robot in free motion [15]-[16] consider the dynamics of the robot to be

$$
\mathbf{M}(\mathbf{q}) \ddot{\mathbf{q}}+\mathbf{C}(\mathbf{q}) \dot{\mathbf{q}}+\mathbf{G}(\mathbf{q})=\boldsymbol{\tau}
$$

in which there is no term involving the interaction force between the robot and the environment. The situation where the robot is in contact with an environment was considered in [17] yet both position and force sensors were needed in the controller, which is expensive to realize in practice.

\section{A. Adaptive Position-error-based Teleoperation}

We extend the control for a single robot to position-error based (PEB) control of a bilateral teleoperator coupled to a human operator and an environment. The principle of PEB control [19] is that it tries to minimize the difference between the master and the slave positions, thus reflecting a force proportional to this difference to the operator once the slave makes contact with an object. The PEB control merit is its simplicity and not requiring force sensors.

To achieve stable and transparent teleoperation, the goals of our PEB-based adaptive control are as follows:

1) Ensure boundedness of signals in the teleoperation system irrespective of dynamic and kinematic uncertainties.

2) Ensure convergence of the position tracking error between the master and the slave to zero.

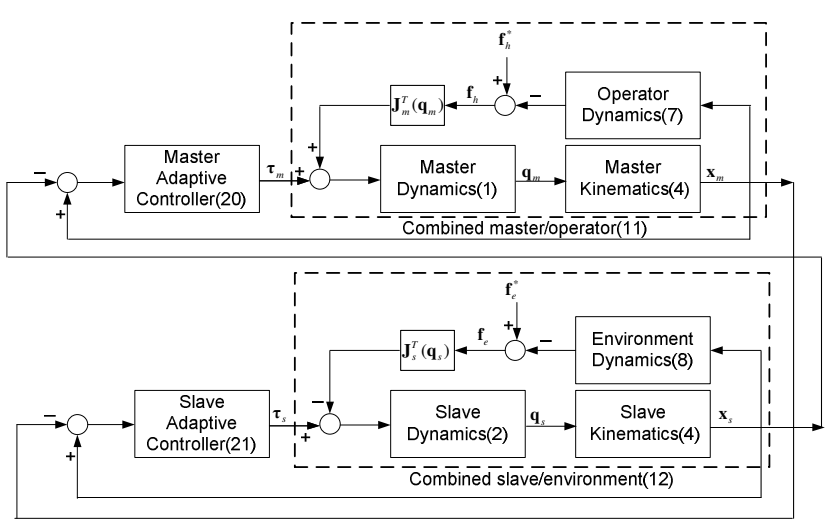

Fig. 1. Structure of adaptive PEB teleoperation control

The structure of our PEB-based adaptive control scheme for teleoperation is shown in Fig. 1. Adaptive controllers are 
designed for the master/operator system and for the slave/environment system, where the dynamics of the master, the slave, the human, and the environment are uncertain, and also the kinematics of the master and the slave are uncertain. In PEB control, the position of the master is the reference position for the slave and vice versa.

\section{B. Teleoperation Control under Dynamic and Kinematic Uncertainties}

When the Jacobian matrices of the master and the slave experience parametric uncertainties, (4) becomes

$$
\begin{aligned}
& \hat{\dot{\mathbf{x}}}_{\mathrm{m}}=\hat{\mathbf{J}}_{\mathrm{m}}\left(\mathbf{q}_{\mathrm{m}}, \widehat{\boldsymbol{\theta}}_{\mathrm{mk}}\right) \dot{\mathbf{q}}_{\mathrm{m}}=\mathbf{Y}_{\mathrm{mk}}\left(\mathbf{q}_{\mathrm{m}}, \dot{\mathbf{q}}_{\mathrm{m}}\right) \widehat{\boldsymbol{\theta}}_{\mathrm{mk}} \\
& \hat{\dot{\mathbf{x}}}_{\mathrm{s}}=\hat{\mathbf{J}}_{\mathrm{s}}\left(\mathbf{q}_{\mathrm{s}}, \widehat{\boldsymbol{\theta}}_{\mathrm{sk}}\right) \dot{\mathbf{q}}_{\mathrm{s}}=\mathbf{Y}_{\mathrm{sk}}\left(\mathbf{q}_{\mathrm{s}}, \dot{\mathbf{q}}_{\mathrm{s}}\right) \widehat{\boldsymbol{\theta}}_{\mathrm{sk}}
\end{aligned}
$$

where $\hat{\mathbf{x}}_{\mathrm{m}}$ and $\hat{\mathbf{x}}_{\mathrm{s}}$ are estimations of $\dot{\mathbf{x}}_{\mathrm{m}}$ and $\dot{\mathbf{x}}_{\mathrm{s}}$, $\hat{\mathbf{J}}_{\mathrm{m}}\left(\mathbf{q}_{\mathrm{m}}, \widehat{\boldsymbol{\theta}}_{\mathrm{mk}}\right)$ and $\hat{\mathbf{J}}_{\mathrm{s}}\left(\mathbf{q}_{\mathrm{s}}, \widehat{\boldsymbol{\theta}}_{\mathrm{sk}}\right)$ are estimations of $\mathbf{J}_{\mathrm{m}}\left(\mathbf{q}_{\mathrm{m}}\right)$ and $\mathbf{J}_{\mathrm{m}}\left(\mathbf{q}_{\mathrm{m}}\right)$, and $\widehat{\boldsymbol{\theta}}_{\mathrm{mk}}$ and $\widehat{\boldsymbol{\theta}}_{\mathrm{sk}}$ are estimations of $\boldsymbol{\theta}_{\mathrm{mk}}$ and $\boldsymbol{\theta}_{\mathrm{sk}}$, respectively. Define two new vectors in joint space for the master side and the slave side:

$$
\dot{\mathbf{q}}_{\mathrm{mr}}=\hat{\mathbf{J}}_{\mathrm{m}}^{-1}\left(\mathbf{q}_{\mathrm{m}}, \widehat{\boldsymbol{\theta}}_{\mathrm{mk}}\right) \dot{\mathbf{x}}_{\mathrm{mr}}, \dot{\mathbf{q}}_{\mathrm{sr}}=\hat{\mathbf{J}}_{\mathrm{s}}^{-1}\left(\mathbf{q}_{\mathrm{s}}, \widehat{\boldsymbol{\theta}}_{\mathrm{sk}}\right) \dot{\mathbf{x}}_{\mathrm{sr}}
$$

where $\hat{\mathbf{J}}_{\mathrm{m}}^{-1}\left(\mathbf{q}_{\mathrm{m}}, \widehat{\boldsymbol{\theta}}_{\mathrm{mk}}\right)$ and $\hat{\mathbf{J}}_{\mathrm{s}}^{-1}\left(\mathbf{q}_{\mathrm{s}}, \widehat{\boldsymbol{\theta}}_{\mathrm{sk}}\right)$ are the inverses of $\hat{\mathbf{J}}_{\mathrm{m}}\left(\mathbf{q}_{\mathrm{m}}, \widehat{\boldsymbol{\theta}}_{\mathrm{mk}}\right)$ and $\hat{\mathbf{J}}_{\mathrm{s}}\left(\mathbf{q}_{\mathrm{s}}, \widehat{\boldsymbol{\theta}}_{\mathrm{sk}}\right)$, respectively. Here,

$$
\dot{\mathbf{x}}_{\mathrm{mr}}=\dot{\mathbf{x}}_{\mathrm{s}}-\alpha \Delta \mathbf{x}_{\mathrm{m}}, \dot{\mathbf{x}}_{\mathrm{sr}}=\dot{\mathbf{x}}_{\mathrm{m}}-\alpha \Delta \mathbf{x}_{\mathrm{s}}
$$

where $\alpha$ is a positive constant, $\Delta \mathbf{x}_{\mathrm{m}}=\mathbf{x}_{\mathrm{m}}-\mathbf{x}_{\mathrm{s}}$, and $\Delta \mathbf{x}_{\mathrm{s}}=\mathbf{x}_{\mathrm{s}}-\mathbf{x}_{\mathrm{m}}$. Also, define two adaptive sliding vectors in joint space for the master side and the slave side as

$$
\mathbf{s}_{\mathrm{m}}=\dot{\mathbf{q}}_{\mathrm{m}}-\dot{\mathbf{q}}_{\mathrm{mr}}, \mathbf{s}_{\mathrm{s}}=\dot{\mathbf{q}}_{\mathrm{s}}-\dot{\mathbf{q}}_{\mathrm{sr}}
$$

Using (17) and Property 1, (11)-(12) become

$$
\begin{aligned}
& \mathbf{M}_{\mathrm{m}}\left(\mathbf{q}_{\mathrm{m}}\right) \dot{\mathbf{s}}_{\mathrm{m}}+\mathbf{C}_{\mathrm{m}}\left(\mathbf{q}_{\mathrm{m}}, \dot{\mathbf{q}}_{\mathrm{m}}\right) \mathbf{s}_{\mathrm{m}}+\mathbf{Y}_{\mathrm{md}} \boldsymbol{\theta}_{\mathrm{md}}=\boldsymbol{\tau}_{\mathrm{m}} \\
& \mathbf{M}_{\mathrm{s}}\left(\mathbf{q}_{\mathrm{s}}\right) \dot{\mathbf{s}}_{\mathrm{s}}+\mathbf{C}_{\mathrm{s}}\left(\mathbf{q}_{\mathrm{s}}, \dot{\mathbf{q}}_{\mathrm{s}}\right) \mathbf{s}_{\mathrm{s}}+\mathbf{Y}_{\mathrm{sd}} \boldsymbol{\theta}_{\mathrm{sd}}=\boldsymbol{\tau}_{\mathrm{s}}
\end{aligned}
$$

where

$$
\mathbf{Y}_{\mathrm{md}} \boldsymbol{\theta}_{\mathrm{md}}=\mathbf{M}_{\mathrm{m}}\left(\mathbf{q}_{\mathrm{m}}\right) \ddot{\mathbf{q}}_{\mathrm{mr}}+\mathbf{C}_{\mathrm{m}}\left(\mathbf{q}_{\mathrm{m}}, \dot{\mathbf{q}}_{\mathrm{m}}\right) \dot{\mathbf{q}}_{\mathrm{mr}}+\mathbf{G}_{\mathrm{m}}\left(\mathbf{q}_{\mathrm{m}}\right)
$$

and

$$
\mathbf{Y}_{\mathrm{sd}} \boldsymbol{\theta}_{\mathrm{sd}}=\mathbf{M}_{\mathrm{s}}\left(\mathbf{q}_{\mathrm{s}}\right) \ddot{\mathbf{q}}_{\mathrm{sr}}+\mathbf{C}_{\mathrm{s}}\left(\mathbf{q}_{\mathrm{s}}, \dot{\mathbf{q}}_{\mathrm{s}}\right) \dot{\mathbf{q}}_{\mathrm{sr}}+\mathbf{G}_{\mathrm{s}}\left(\mathbf{q}_{\mathrm{s}}\right) .
$$

Inspired by [15]-[17], we are now in a position to propose our nonlinear adaptive bilateral control algorithm. The algorithm is composed of the following three parts:

- Control laws for the master and the slave:

$$
\begin{aligned}
& \boldsymbol{\tau}_{\mathrm{m}}=\mathbf{Y}_{\mathrm{md}} \widehat{\boldsymbol{\theta}}_{\mathrm{md}}-\hat{\mathbf{J}}_{\mathrm{m}}^{\mathrm{T}}\left(\mathbf{q}_{\mathrm{m}}, \widehat{\boldsymbol{\theta}}_{\mathrm{mk}}\right) \mathbf{K}_{\mathrm{m}}\left(\Delta \hat{\dot{\mathbf{x}}}_{\mathrm{m}}+\alpha \Delta \mathbf{x}_{\mathrm{m}}\right) \\
& \boldsymbol{\tau}_{\mathrm{s}}=\mathbf{Y}_{\mathrm{sd}} \widehat{\boldsymbol{\theta}}_{\mathrm{sd}}-\hat{\mathbf{J}}_{\mathrm{s}}^{\mathrm{T}}\left(\mathbf{q}_{\mathrm{s}}, \widehat{\boldsymbol{\theta}}_{\mathrm{sk}}\right) \mathbf{K}_{\mathrm{s}}\left(\Delta \hat{\mathbf{x}}_{\mathrm{s}}+\alpha \Delta \mathbf{x}_{\mathrm{s}}\right)
\end{aligned}
$$

where $\mathbf{K}_{\mathrm{m}}$ and $\mathbf{K}_{\mathrm{s}}$ are symmetric positive definite matrices, $\Delta \hat{\dot{\mathbf{x}}}_{\mathrm{m}}=\hat{\dot{\mathbf{x}}}_{\mathrm{m}}-\dot{\mathbf{x}}_{\mathrm{s}}, \Delta \hat{\dot{\mathbf{x}}}_{\mathrm{s}}=\hat{\dot{\mathbf{x}}}_{\mathrm{s}}-\dot{\mathbf{x}}_{\mathrm{m}}, \widehat{\boldsymbol{\theta}}_{\mathrm{md}}$ and $\widehat{\boldsymbol{\theta}}_{\mathrm{sd}}$ are the estimations of $\boldsymbol{\theta}_{\mathrm{md}}$ and $\boldsymbol{\theta}_{\mathrm{sd}}$, respectively.

- Dynamic update laws:

$$
\begin{aligned}
& \dot{\boldsymbol{\theta}}_{\mathrm{md}}=-\mathbf{L}_{\mathrm{md}} \mathbf{Y}_{\mathrm{md}}^{\mathrm{T}} \mathbf{s}_{\mathrm{m}} \\
& \dot{\hat{\boldsymbol{\theta}}}_{\mathrm{sd}}=-\mathbf{L}_{\mathrm{sd}} \mathbf{Y}_{\mathrm{sd}}^{\mathrm{T}} \mathbf{s}_{\mathrm{s}}
\end{aligned}
$$

- Kinematic update laws:

$$
\begin{aligned}
\dot{\hat{\boldsymbol{\theta}}}_{\mathrm{mk}} & =2 \mathbf{L}_{\mathrm{mk}} \mathbf{Y}_{\mathrm{mk}}^{\mathrm{T}}\left(\mathbf{q}_{\mathrm{m}}, \dot{\mathbf{q}}_{\mathrm{m}}\right) \mathbf{K}_{\mathrm{m}}\left(\Delta \dot{\mathbf{x}}_{\mathrm{m}}+\alpha \Delta \mathbf{x}_{\mathrm{m}}\right) \\
\dot{\hat{\boldsymbol{\theta}}}_{\mathrm{sk}} & =2 \mathbf{L}_{\mathrm{sk}} \mathbf{Y}_{\mathrm{sk}}^{\mathrm{T}}\left(\mathbf{q}_{\mathrm{s}}, \dot{\mathbf{q}}_{\mathrm{s}}\right) \mathbf{K}_{\mathrm{s}}\left(\Delta \dot{\mathbf{x}}_{\mathrm{s}}+\alpha \Delta \mathbf{x}_{\mathrm{s}}\right)
\end{aligned}
$$

where $\mathbf{L}_{\mathrm{mk}}, \mathbf{L}_{\mathrm{md}}, \mathbf{L}_{\mathrm{sk}}$ and $\mathbf{L}_{\mathrm{sd}}$ are all symmetric positive definite but otherwise arbitrary matrices.

Each of the control laws (20)-(21) includes two parts. The first part is feedforward model-based estimation and the second part involves feedback compensation for velocity and position tracking. Substituting (20)-(21) into (18)-(19), the closed-loop teleoperation system is obtained as

$$
\begin{gathered}
\mathbf{M}_{\mathrm{m}}\left(\mathbf{q}_{\mathrm{m}}\right) \dot{\mathbf{s}}_{\mathrm{m}}+\mathbf{C}_{\mathrm{m}}\left(\mathbf{q}_{\mathrm{m}}, \dot{\mathbf{q}}_{\mathrm{m}}\right) \mathbf{s}_{\mathrm{m}}+\mathbf{Y}_{\mathrm{md}} \Delta \boldsymbol{\theta}_{\mathrm{md}} \\
\quad+\hat{\mathbf{J}}_{\mathrm{m}}^{\mathrm{T}}\left(\mathbf{q}_{\mathrm{m}}, \widehat{\boldsymbol{\theta}}_{\mathrm{mk}}\right) \mathbf{K}_{\mathrm{m}}\left(\Delta \hat{\dot{\mathbf{x}}}_{\mathrm{m}}+\alpha \Delta \mathbf{x}_{\mathrm{m}}\right)=\mathbf{0} \\
\mathbf{M}_{\mathrm{s}}\left(\mathbf{q}_{\mathrm{s}}\right) \dot{\mathbf{s}}_{\mathrm{s}}+\mathbf{C}_{\mathrm{s}}\left(\mathbf{q}_{\mathrm{s}}, \dot{\mathbf{q}}_{\mathrm{s}}\right) \mathbf{s}_{\mathrm{s}}+\mathbf{Y}_{\mathrm{sd}} \Delta \boldsymbol{\theta}_{\mathrm{sd}} \\
\quad+\hat{\mathbf{J}}_{\mathrm{s}}^{\mathrm{T}}\left(\mathbf{q}_{\mathrm{s}}, \widehat{\boldsymbol{\theta}}_{\mathrm{sk}}\right) \mathbf{K}_{\mathrm{s}}\left(\Delta \hat{\dot{\mathbf{x}}}_{\mathrm{s}}+\alpha \Delta \mathbf{x}_{\mathrm{s}}\right)=\mathbf{0}
\end{gathered}
$$

where $\Delta \boldsymbol{\theta}_{\mathrm{md}}=\boldsymbol{\theta}_{\mathrm{md}}-\widehat{\boldsymbol{\theta}}_{\mathrm{md}}$ and $\Delta \boldsymbol{\theta}_{\mathrm{sd}}=\boldsymbol{\theta}_{\mathrm{sd}}-\widehat{\boldsymbol{\theta}}_{\mathrm{sd}}$.

Remark 2: In the control laws (20)-(21), it is assumed that $\mathbf{x}_{\mathrm{m}}, \mathbf{x}_{\mathrm{s}}, \mathbf{q}_{\mathrm{m}}$ and $\mathbf{q}_{\mathrm{s}}$ can be measured from position sensors. Differentiating them for finding $\mathbf{J}_{\mathrm{m}}$ and $\mathbf{J}_{\mathrm{s}}$, however, will involve inaccuracies due to noise. This is the reason for explicitly considering kinematic uncertainties and thus developing kinematics update laws in this paper.

Remark 3: The vector $\boldsymbol{\theta}_{\mathrm{md}}$ includes unknown dynamic parameters of the master and the human operator. Similarly, $\boldsymbol{\theta}_{\text {sd }}$ includes the unknown dynamic parameters of the slave and the environment. Here, $\mathbf{f}_{\mathrm{h}}^{*}$ is treated as an unknown system parameter and is included in $\boldsymbol{\theta}_{\mathrm{md}}$. Also, $\mathbf{f}_{\mathrm{e}}^{*}$ is included in $\boldsymbol{\theta}_{\mathrm{sd}}$. Such an approach is effective when $\mathbf{f}_{\mathrm{h}}^{*}$ and $\mathbf{f}_{\mathrm{e}}^{*}$ change slowly with respect to time.

Theorem: Consider the nonlinear teleoperation system (11)(12), which is the result of the dynamics of the human operator (7) and the environment (8) being incorporated into the master robot (1) and the slave robot (2). If this teleoperation system is controlled by (20)-(21) using the dynamic update laws (22)-(23) and the kinematic update laws (24)-(25), then all the signals in the closed-loop system are bounded.

The proof of the Theorem and a discussion on the position tracking performance can be found in Appendix.

\section{Simulation STUdY}

In this section, simulations are conducted to illustrate the performance of the proposed controller. Both the master and the slave are considered to be two-link, revolute-joint planar robots [18] with similar dynamics and kinematics.

As for the operator and the environment, let us take

$$
\begin{aligned}
\mathbf{M}_{\mathrm{h}} & =\mathrm{m}_{\mathrm{h}} \mathbf{I}, \mathbf{B}_{\mathrm{h}}=\mathrm{b}_{\mathrm{h}} \mathbf{I}, \mathbf{K}_{\mathrm{h}}=\mathrm{k}_{\mathrm{h}} \mathbf{I}, \\
\mathbf{M}_{\mathrm{e}} & =\mathrm{m}_{\mathrm{e}} \mathbf{I}, \mathbf{B}_{\mathrm{e}}=\mathrm{b}_{\mathrm{e}} \mathbf{I}, \mathbf{K}_{\mathrm{e}}=\mathrm{k}_{\mathrm{e}} \mathbf{I},
\end{aligned}
$$


where $\mathrm{m}_{\mathrm{h}}, \mathrm{m}_{\mathrm{e}}, \mathrm{b}_{\mathrm{h}}, \mathrm{b}_{\mathrm{e}}, \mathrm{k}_{\mathrm{h}}$ and $\mathrm{k}_{\mathrm{e}}$ are the mass, damping, and stiffness coefficients of the operator's hand and the environment, respectively. Let $\mathbf{f}_{\mathrm{h}}^{*}$ starts from a zero value, $\mathbf{f}_{\mathrm{h}}^{*}=\left[\mathrm{f}_{\mathrm{h} 1}^{*}, 0\right]=[25(1-\cos 0.1 \mathrm{t}), 0]^{\mathrm{T}}$. Also, take $\mathbf{f}_{\mathrm{e}}^{*}=[0,0]$.

For simplicity, gravity is ignored. Then, the unknown dynamic parameter vectors can be expressed as

$$
\begin{gathered}
\boldsymbol{\theta}_{\mathrm{md}}=\left[\mathrm{l}_{2}^{2}\left(\mathrm{~m}_{2}+\mathrm{m}_{\mathrm{h}}\right), \mathrm{l}_{1} \mathrm{l}_{2} \mathrm{~m}_{2}, \mathrm{l}_{1} \mathrm{l}_{2} \mathrm{~m}_{\mathrm{h}}, \mathrm{l}_{1} \mathrm{l}_{2} \mathrm{~b}_{\mathrm{h}}, \mathrm{l}_{2}^{2} \mathrm{~b}_{\mathrm{h}}, \mathrm{l}_{1} \mathrm{l}_{2} \mathrm{k}_{\mathrm{h}}\right. \\
\left.\mathrm{l}_{1}^{2}\left(\mathrm{~m}_{1}+\mathrm{m}_{2}+\mathrm{m}_{\mathrm{h}}\right), \mathrm{l}_{1}^{2} \mathrm{~b}_{\mathrm{h}}, \mathrm{f}_{\mathrm{h} 1}^{*} \mathrm{l}_{1}, \mathrm{f}_{\mathrm{h} 1}^{*} \mathrm{l}_{2}\right]^{\mathrm{T}} \\
\boldsymbol{\theta}_{\mathrm{sd}}=\left[\mathrm{l}_{2}^{2}\left(\mathrm{~m}_{2}+\mathrm{m}_{\mathrm{e}}\right), \mathrm{l}_{1} \mathrm{l}_{2} \mathrm{~m}_{2}, \mathrm{l}_{1} \mathrm{l}_{2} \mathrm{~m}_{\mathrm{e}}, \mathrm{l}_{1} \mathrm{l}_{2} \mathrm{~b}_{\mathrm{e}}, \mathrm{l}_{2}^{2} \mathrm{~b}_{\mathrm{e}}, \mathrm{l}_{1} \mathrm{l}_{2} \mathrm{k}_{\mathrm{e}}\right. \\
\left.\mathrm{l}_{1}^{2}\left(\mathrm{~m}_{1}+\mathrm{m}_{2}+\mathrm{m}_{\mathrm{e}}\right), \mathrm{l}_{1}^{2} \mathrm{~b}_{\mathrm{e}}\right]^{\mathrm{T}}
\end{gathered}
$$

where $l_{1}$ and $l_{2}$ are the lengths of the links, and $m_{1}$ and $m_{2}$ are the point masses of the links. The dynamic regressor matrices $\mathbf{Y}_{\mathrm{md}}$ and $\mathbf{Y}_{\mathrm{sd}}$ are obtained based on Property 1 .

As far as kinematics, the robot Jacobian matrix is

$$
\mathbf{J}(\mathbf{q})=\left[\begin{array}{cc}
-\mathrm{l}_{1} \mathrm{~s}_{1}-\mathrm{l}_{2} \mathrm{~s}_{12} & -\mathrm{l}_{2} \mathrm{~s}_{12} \\
\mathrm{l}_{1} \mathrm{c}_{1}+\mathrm{l}_{2} \mathrm{c}_{12} & \mathrm{l}_{2} \mathrm{c}_{12}
\end{array}\right]
$$

Then, the unknown kinematic parameter vectors can be expressed as $\boldsymbol{\theta}_{\mathrm{mk}}=\boldsymbol{\theta}_{\mathrm{sk}}=\left[\mathrm{l}_{1}, \mathrm{l}_{2}\right]^{\mathrm{T}}$ and the kinematic regressor matrices $\mathbf{Y}_{\mathrm{mk}}\left(\mathbf{q}_{\mathrm{m}}, \dot{\mathbf{q}}_{\mathrm{m}}\right)$ and $\mathbf{Y}_{\mathrm{sk}}\left(\mathbf{q}_{\mathrm{s}}, \dot{\mathbf{q}}_{\mathrm{s}}\right)$ are obtained based on Property 3.

In the simulation, the parameters of the robots [18], the operator and the environment [13] are given in Table I.

TABLE I

Model Parameters in THE Simulations

\begin{tabular}{cccc}
\hline \hline $\mathrm{I}_{1}, \mathrm{l}_{2}$ & $\mathrm{~m}_{1}$ & $\mathrm{~m}_{2}$ & $\mathrm{~m}_{\mathrm{h}}$ \\
$0.5(\mathrm{~m})$ & $4.6(\mathrm{~kg})$ & $2.3(\mathrm{~kg})$ & $0.2(\mathrm{~kg})$ \\
$\mathrm{b}_{\mathrm{h}}$ & $\mathrm{k}_{\mathrm{h}}$ & $\mathrm{m}_{\mathrm{e}}$ & $\mathrm{b}_{\mathrm{e}}$ \\
$50\left(\mathrm{Nsm}^{-1}\right)$ & $1000\left(\mathrm{Nm}^{-1}\right)$ & $0.1(\mathrm{~kg})$ & $20\left(\mathrm{Nsm}^{-1}\right)$ \\
$\mathrm{k}_{\mathrm{e}}$ & $\mathbf{K}_{\mathrm{m}}$ & $\mathrm{L}_{\mathrm{md}}$ & $\mathrm{L}_{\mathrm{mk}}$ \\
$1000\left(\mathrm{Nm}^{-1}\right)$ & $200 \mathrm{I}$ & $40 \mathrm{I}$ & $10 \mathrm{I}$ \\
$\alpha$ & $\mathrm{K}_{\mathrm{s}}$ & $\mathrm{L}_{\mathrm{sd}}$ & $\mathrm{L}_{\mathrm{sk}}$ \\
0.25 & $200 \mathrm{I}$ & $40 \mathrm{I}$ & $10 \mathrm{I}$ \\
\hline \hline
\end{tabular}

According to Table 1, the actual parameter vectors are

$$
\begin{aligned}
\boldsymbol{\theta}_{\mathrm{md}}= & {[0.625,0.575,0.05,12.5,12.5,250,1.775,12.5,} \\
& 17.5(1-\cos 0.1 \mathrm{t}), 17.5(1-\cos 0.1 \mathrm{t})]^{\mathrm{T}}, \\
\boldsymbol{\theta}_{\mathrm{sd}}= & {[0.6,0.575,0.025,5,5,250,1.75,5]^{\mathrm{T}}, } \\
\boldsymbol{\theta}_{\text {sd }}= & \boldsymbol{\theta}_{\text {sk }}=[0.5,0.5]^{\mathrm{T}} .
\end{aligned}
$$

In the simulations, the initial values were randomly set as

$$
\begin{aligned}
& \mathbf{x}_{\mathrm{m}}(0)=\mathbf{x}_{\mathrm{s}}(0)=[0.6,0.2]^{\mathrm{T}}, \\
& \widehat{\boldsymbol{\theta}}_{\mathrm{md}}(0)=[0.5,0.6,0.1,11,13,240,1,12,13,10]^{\mathrm{T}}, \\
& \widehat{\boldsymbol{\theta}}_{\mathrm{sd}}(0)=[0.3,0.5,0.02,6,6,240,2,4]^{\mathrm{T}}, \\
& \widehat{\boldsymbol{\theta}}_{\mathrm{mk}}(0)=\widehat{\boldsymbol{\theta}}_{\mathrm{sk}}(0)=[1,1]^{\mathrm{T}} .
\end{aligned}
$$

The transparency of the proposed control approach is compared with the conventional adaptive control approach, which cannot deal with kinematic uncertainties. The simulation results are shown in Fig. 2-3. Using the proposed control scheme, the slave tracks the position of the master well, while in the conventional adaptive control scheme the position tracking error is clearly larger. As for force tracking, although there are errors both in the proposed control scheme and the conventional adaptive one, the error in the proposed control scheme is smaller at certain points. This is reasonable because the proposed controller is based on the PEB architecture and not on the direct force reflection architecture or the 4-channel architecture, which can achieve better forcing tracking. Since $\mathbf{f}_{\mathrm{h}}^{*}$ is zero in the $\mathrm{Y}$ axis, the position and force are not shown in that direction.
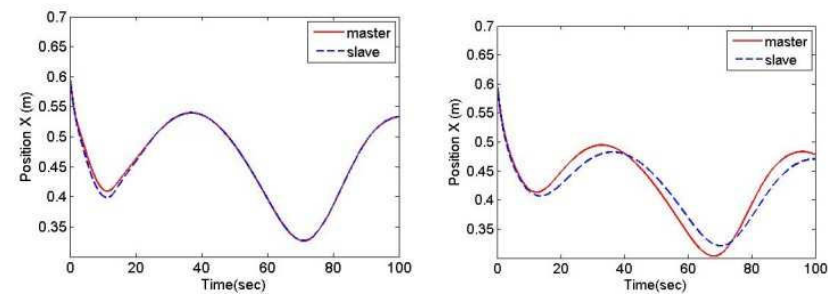

Fig. 2. Master and slave positions $\left(\mathrm{x}_{\mathrm{m}}\right.$ and $\left.\mathrm{x}_{\mathrm{s}}\right)$ in $\mathrm{X}$ axis: (left) proposed adaptive control, (right) conventional adaptive control
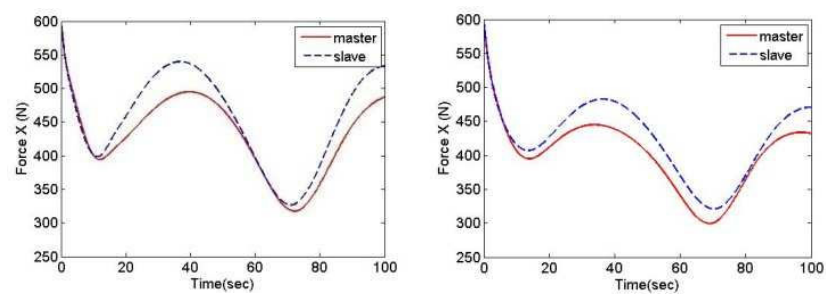

Fig. 3. Master and slave forces $\left(f_{h}\right.$ and $f_{e}$ ) in $X$ axis:

(left) proposed adaptive control, (right) conventional adaptive control

It is worth noting that a key point in adaptive control is that the tracking error should converge regardless of whether the input is persistently exciting or not, i.e., one does not need parameter convergence for tracking error convergence.

\section{CONCLUDING REMARKS}

In this work, we presented a nonlinear adaptive algorithm to cope with uncertainties in the dynamics and kinematics of a teleoperation system. The dynamics of the human operator and the environment were incorporated into the master and the slave dynamics, respectively. The adaptive controllers designed for the master and the slave do not need any force measurements. The stability of the closed-loop system is guaranteed and the convergence of the position tracking error is discussed. To the best of our knowledge, this is the first time that both dynamic and kinematic uncertainties have been considered in control of a teleoperation system.

The proposed controller is based on the PEB architecture, which has a limitation in terms of force tracking compared to the direct force reflection architecture and the 4-channel architecture [19]. Addressing this and accounting for variations in the communication time delay and nonlinear terms such as joint friction remain as future work. 


\section{APPENDIX}

Stability and position convergence analysis:

Consider a unified Lyapunov candidate function as

$$
\mathrm{V}=\mathrm{V}_{1}+\mathrm{V}_{2}
$$

where $V_{1}$ and $V_{2}$ are the Lyapunov functions for a single robot [15]-[17]:

$$
\begin{aligned}
\mathrm{V}_{1}= & \frac{1}{2} \mathbf{s}_{\mathrm{m}}^{\mathrm{T}} \mathbf{M}_{\mathrm{m}}\left(\mathbf{q}_{\mathrm{m}}\right) \mathbf{s}_{\mathrm{m}}+\alpha \Delta \mathbf{x}_{\mathrm{m}}^{\mathrm{T}} \mathbf{K}_{\mathrm{m}} \Delta \mathbf{x}_{\mathrm{m}}+\frac{1}{2} \Delta \boldsymbol{\theta}_{\mathrm{md}}^{\mathrm{T}} \mathbf{L}_{\mathrm{md}}^{-1} \Delta \boldsymbol{\theta}_{\mathrm{md}} \\
& +\frac{1}{2} \Delta \boldsymbol{\theta}_{\mathrm{mk}}^{\mathrm{T}} \mathbf{L}_{\mathrm{mk}}^{-1} \Delta \boldsymbol{\theta}_{\mathrm{mk}} \\
\mathrm{V}_{2}= & \frac{1}{2} \mathbf{s}_{\mathrm{s}}^{\mathrm{T}} \mathbf{M}_{\mathrm{s}}\left(\mathbf{q}_{\mathrm{s}}\right) \mathbf{s}_{\mathrm{s}}+\alpha \Delta \mathbf{x}_{\mathrm{s}}^{\mathrm{T}} \mathbf{K}_{\mathrm{s}} \Delta \mathbf{x}_{\mathrm{s}}+\frac{1}{2} \Delta \boldsymbol{\theta}_{\mathrm{sd}}^{\mathrm{T}} \mathbf{L}_{\mathrm{sd}}^{-1} \Delta \boldsymbol{\theta}_{\mathrm{sd}} \\
& +\frac{1}{2} \Delta \boldsymbol{\theta}_{\mathrm{sk}}^{\mathrm{T}} \mathbf{L}_{\mathrm{sk}}^{-1} \Delta \boldsymbol{\theta}_{\mathrm{sk}}
\end{aligned}
$$

where $\Delta \boldsymbol{\theta}_{\mathrm{mk}}=\boldsymbol{\theta}_{\mathrm{mk}}-\widehat{\boldsymbol{\theta}}_{\mathrm{mk}}$, and $\Delta \boldsymbol{\theta}_{\mathrm{sk}}=\boldsymbol{\theta}_{\mathrm{sk}}-\widehat{\boldsymbol{\theta}}_{\mathrm{sk}}$. Since $\mathbf{M}_{\mathrm{m}}\left(\mathbf{q}_{\mathrm{m}}\right), \mathbf{M}_{\mathrm{s}}\left(\mathbf{q}_{\mathrm{s}}\right), \mathbf{K}_{\mathrm{m}}, \mathbf{K}_{\mathrm{s}}, \mathbf{L}_{\mathrm{md}}, \mathbf{L}_{\mathrm{sd}}, \mathbf{L}_{\mathrm{mk}}$ and $\mathbf{L}_{\mathrm{sk}}$ are all positive definite, $\mathrm{V}$ is positive definite.

Using Property 4, the derivative of $\mathrm{V}$ along the trajectory of the closed-loop system (26)-(27) becomes

$$
\begin{aligned}
\dot{V}= & -\mathbf{K}_{\mathrm{m}}\left(\Delta \dot{\mathbf{x}}_{\mathrm{m}}^{\mathrm{T}} \Delta \dot{\mathbf{x}}_{\mathrm{m}}+\alpha^{2} \Delta \mathbf{x}_{\mathrm{m}}^{\mathrm{T}} \Delta \mathbf{x}_{\mathrm{m}}\right. \\
& \left.+\Delta \boldsymbol{\theta}_{\mathrm{mk}}^{\mathrm{T}} \mathbf{Y}_{\mathrm{mk}}^{\mathrm{T}}\left(\mathbf{q}_{\mathrm{m}}, \dot{\mathbf{q}}_{\mathrm{m}}\right) \mathbf{Y}_{\mathrm{mk}}\left(\mathbf{q}_{\mathrm{m}}, \dot{\mathbf{q}}_{\mathrm{m}}\right) \Delta \boldsymbol{\theta}_{\mathrm{mk}}\right) \\
& -\mathbf{K}_{\mathrm{s}}\left(\Delta \dot{\mathbf{x}}_{\mathrm{s}}^{\mathrm{T}} \Delta \dot{\mathbf{x}}_{\mathrm{s}}+\alpha^{2} \Delta \mathbf{x}_{\mathrm{s}}^{\mathrm{T}} \Delta \mathbf{x}_{\mathrm{s}}\right. \\
& \left.+\Delta \boldsymbol{\theta}_{\mathrm{sk}}^{\mathrm{T}} \mathbf{Y}_{\mathrm{sk}}^{\mathrm{T}}\left(\mathbf{q}_{\mathrm{s}}, \dot{\mathbf{q}}_{\mathrm{s}}\right) \mathbf{Y}_{\mathrm{sk}}\left(\mathbf{q}_{\mathrm{s}}, \dot{\mathbf{q}}_{\mathrm{s}}\right) \Delta \boldsymbol{\theta}_{\mathrm{sk}}\right) \\
& -\mathbf{s}_{\mathrm{m}}^{\mathrm{T}}\left(\mathbf{J}_{\mathrm{m}}^{\mathrm{T}}\left(\mathbf{q}_{\mathrm{m}}\right) \mathbf{B}_{\mathrm{h}} \mathbf{J}_{\mathrm{m}}\left(\mathbf{q}_{\mathrm{m}}\right)\right) \mathbf{s}_{\mathrm{m}} \\
& -\mathbf{s}_{\mathrm{s}}^{\mathrm{T}}\left(\mathbf{J}_{\mathrm{s}}^{\mathrm{T}}\left(\mathbf{q}_{\mathrm{s}}\right) \mathbf{B}_{\mathrm{e}} \mathbf{J}_{\mathrm{s}}\left(\mathbf{q}_{\mathrm{s}}\right)\right) \mathbf{s}_{\mathrm{s}}
\end{aligned}
$$

Note that $\mathbf{K}_{\mathrm{m}}, \mathbf{K}_{\mathrm{s}}, \mathbf{B}_{\mathrm{h}}$ and $\mathrm{B}_{\mathrm{e}}$ are positive definite matrices. Therefore, $\dot{V}$ is negative semi-definite. The reason for $\dot{V}$ not being negative definite is that for $\mathbf{s}_{\mathrm{m}}=\mathbf{s}_{\mathrm{s}}=\Delta \mathbf{x}_{\mathrm{m}}=\Delta \mathbf{x}_{\mathrm{s}}=$ $\Delta \boldsymbol{\theta}_{\mathrm{mk}}=\Delta \boldsymbol{\theta}_{\mathrm{sk}}=\mathbf{0}$, but $\Delta \boldsymbol{\theta}_{\mathrm{md}} \neq \mathbf{0}$ or $\Delta \boldsymbol{\theta}_{\mathrm{sd}} \neq \mathbf{0}$, we have $\dot{\mathrm{V}}=0$. Therefore, $\mathrm{V}$ is bounded and all the signals including $\mathbf{s}_{\mathrm{m}}, \mathbf{s}_{\mathrm{s}}, \Delta \mathbf{x}_{\mathrm{m}}, \Delta \mathbf{x}_{\mathrm{s}}, \Delta \boldsymbol{\theta}_{\mathrm{md}}, \Delta \boldsymbol{\theta}_{\mathrm{sd}}, \Delta \boldsymbol{\theta}_{\mathrm{mk}}$ and $\Delta \boldsymbol{\theta}_{\mathrm{sk}}$ are bounded.

As far as position error convergence, we know that $\Delta \mathbf{x}_{\mathrm{S}}$ is bounded. Integrating $\dot{V}$ gives us

$$
\begin{aligned}
\mathrm{V}(\mathrm{t})=\int_{0}^{t} \dot{\mathrm{V}} \mathrm{dt}= & -\int_{0}^{t} \mathbf{K}_{\mathrm{m}}\left(\left\|\Delta \dot{\mathbf{x}}_{\mathrm{m}}\right\|^{2}+\alpha^{2}\left\|\Delta \mathbf{x}_{\mathrm{m}}\right\|^{2}\right. \\
& \left.+\left\|\mathbf{Y}_{\mathrm{mk}}\left(\mathbf{q}_{\mathrm{m}}, \dot{\mathbf{q}}_{\mathrm{m}}\right) \Delta \boldsymbol{\theta}_{\mathrm{mk}}\right\|^{2}\right) \mathrm{dt} \\
& -\int_{0}^{t} \mathbf{K}_{\mathrm{s}}\left(\left\|\Delta \dot{\mathbf{x}}_{\mathrm{s}}\right\|^{2}+\alpha^{2}\left\|\Delta \mathbf{x}_{\mathrm{s}}\right\|^{2}\right. \\
& \left.+\left\|\mathbf{Y}_{\mathrm{sk}}\left(\mathbf{q}_{\mathrm{s}}, \dot{\mathbf{q}}_{\mathrm{s}}\right) \Delta \boldsymbol{\theta}_{\mathrm{sk}}\right\|^{2}\right) \mathrm{dt} \\
& -\int_{0}^{t}\left(\mathbf{B}_{\mathbf{h}} \mathbf{s}_{\mathrm{m}}^{\mathrm{T}}\left(\mathbf{J}_{\mathrm{m}}^{\mathrm{T}}\left(\mathbf{q}_{\mathrm{m}}\right) \mathbf{J}_{\mathrm{m}}\left(\mathbf{q}_{\mathrm{m}}\right)\right) \mathbf{s}_{\mathrm{s}}\right. \\
& \left.+\mathbf{B}_{\mathrm{e}} \mathbf{s}_{\mathrm{s}}^{\mathrm{T}}\left(\mathbf{J}_{\mathrm{s}}^{\mathrm{T}}\left(\mathbf{q}_{\mathrm{s}}\right) \mathbf{J}_{\mathrm{s}}\left(\mathbf{q}_{\mathrm{s}}\right)\right) \mathbf{s}_{\mathrm{s}}\right) \mathrm{dt}
\end{aligned}
$$

Since $\mathrm{V}(\mathrm{t})$ is bounded, as $t \rightarrow \infty$, we get that $\int_{0}^{\infty}\left\|\Delta \mathbf{x}_{\mathrm{s}}\right\|^{2} \mathrm{dt}$ and $\int_{0}^{\infty}\left\|\Delta \dot{\mathbf{x}}_{\mathrm{s}}\right\|^{2} \mathrm{dt}$ are bounded (i.e., $\Delta \mathbf{x}_{\mathrm{s}}, \Delta \dot{\mathbf{x}}_{\mathrm{s}} \in \mathrm{L}^{2}$ ). For a robotic manipulator, it is not unreasonable to deduce that $\Delta \dot{\mathbf{x}}_{\mathrm{s}}$ is also bounded. Having $\Delta \mathbf{x}_{\mathrm{s}}$ bounded, $\Delta \dot{\mathbf{x}}_{\mathrm{s}}$ bounded, and $\Delta \mathbf{x}_{\mathrm{s}} \in \mathrm{L}^{2}$ and using Barbalat's lemma [20], we can obtain that $\lim _{t \rightarrow \infty} \Delta \mathbf{x}_{\mathrm{s}}=\lim _{t \rightarrow \infty}\left(\mathbf{x}_{\mathrm{s}}-\mathbf{x}_{\mathrm{m}}\right) \rightarrow \mathbf{0}$.

\section{REFERENCES}

[1] S. Salcudean, "Control for teleoperation and haptic interfaces," Control Problems in Robotics and Automation, Eds. New York: SpringerVerlag, pp. 51-56, 1998.

[2] P. Hokayem and M. Spong, "Bilateral teleoperation: an historical survey," Automatica, vol. 42, no. 12, pp. 2035-2057, 2006.

[3] K.H. Zaad, and S.E., Salcudean, "Adaptive Impedance Reflecting Teleoperation," in Proceedings of the IEEE International Conference on Robotics and Automation, Minnesota, USA, April, pp. 1369-1374, 1996.

[4] Hyoung-Ki Lee and M. J. Chung, "Adaptive controller of a master-slave system for transparent teleoperation," Journal of Robotic systems, vol. 15, no. 8, pp. 465-475, 1998.

[5] M. Shi, G. Tao and H. Liu, "Adaptive control of teleoperation systems," Journal of X-Ray Science and Technology, vol. 10, no. 1-2, pp. 37-57, 2002.

[6] K.B. Fite, M. Goldfarb, and A. Rubio, "Loop Shaping for Transparency and Stability Robustness in Time-delayed Bilateral Telemanipulation," Journal of Dynamic Systems, Measurement and Control, Transactions of the ASME, Vol. 126, no. 3, pp. 650-656, 2004.

[7] J. H. Ryu and D. S. Kwon, "A novel adaptive bilateral control scheme using similar closed-loop dynamic characteristics of master/slave manipulators," Journal of Robotic Systems, vol. 18, no. 9, pp. 533-543, 2001.

[8] N. V. Q. Hung, T. Narikiyo and H. D. Tuan, "Nonlinear adaptive control of master-slave system in teleoperation," Control Engineering Practice, vol. 11, no. 1, pp. 1-10, 2003.

[9] N. Chopra, M. W. Spong and R. Lozano, "Synchronization of bilateral teleoperators with time delay," Automatica, vol. 44, no. 8, pp. 21422148, 2008.

[10] E. Nuño, R. Ortega and L. Basañez, "An adaptive controller for nonlinear teleoperators," Automatica, vol. 46, no. 1, pp. 155-159, 2010.

[11] Wen-Hong Zhu and S. E. Salcudean, "Stability guaranteed teleoperation: an adaptive motion/force control approach," IEEE transactions on automatic control, vol. 45, no. 11, pp. 1951-1969, 2000.

[12] S. Sirouspour and P. Setoodeh, "Adaptive nonlinear teleoperation control in multi-master/multi-slave environments," in Proceeding of the IEEE Conference on Control Applications, Toronto, Canada, August, pp. 1263-1268, 2005.

[13] P. Malysz and S. Sirouspour, "Nonlinear and filtered force/position mapping in bilateral teleoperation with application to enhanced stiffness discrimination," IEEE Transaction on Robotics, vol. 25, no. 5, pp. 1134-1149, 2009.

[14] D. H. Kim, K. H. Cook, J. H. Oh, "Identification and compensation of robot kinematic parameter for positioning accuracy improvement," Robotica, Vol. 9, no. 1, pp. 99-105, 1991.

[15] C. C. Cheah, C. Liu and J. J. E.Slotine, "Adaptive tracking control for robots with unknown kinematic and dynamic properties," The International Journal of Robotics Research, vol.25, no.3, pp. 283-296, 2006.

[16] C. C. Cheah, C. Liu and J. J. E. Slotine, "Adaptive Jacobian tracking control of robots with uncertainties in kinematic, dynamic and actuator models," IEEE transactions on automatic control, vol. 51, no. 6, pp. 1024-1029, 2006.

[17] C. C. Cheah, Y. Zhao and J. J. E. Slotine, "Adaptive Jacobian motion and force tracking control for constrained robots with uncertainties," in Proceeding of the IEEE International Conference on Robotics and Automation, Florida, USA, May, pp. 2226-2231, 2006.

[18] J. Craig, Introduction to robotics: mechanics and control $\left(3^{\text {rd }} \mathrm{Ed}\right.$.), Pearson Prentice Hall, USA, 2005.

[19] M. Tavakoli, A. Aziminejad, R.V. Patel and M. Moallem, "Highfidelity bilateral teleoperation systems and the effect of multimodal haptics," IEEE Transactions on Systems, Man, and Cybernetics - Part $B$, vol. 37, no. 6, pp. 1512-1528, 2007.

[20] J. J. E. Slotine and W. Li, Applied nonlinear control, Prentice-Hall, Englewood Cliffs, NJ, 1991. 